\title{
"Chemical Triangle" as a modern intellectual basis for digital systematization of energy characteristics of substances
}

\author{
Oleg Sirotkin ${ }^{1, *}$, and Rostislav Sirotkin ${ }^{1}$ \\ ${ }^{1}$ School of Materials Science and Engineering, Kazan State Power Engineering University, Russia
}

\begin{abstract}
It was shown that sustainable power and mechanical engineering relies primarily on chemical transformation of matter, since a chemical substance (in the form of homo- and heteronuclear compounds of elements) is the most accessible type of substance on Earth. As a result, low-, oligo- and high-molecular and non-molecular (metallic and ionic) chemicals and products (fuels, polymers, alloys, glasses, etc.) are primary raw materials for production of thermal and electrical energy, as well as materials needed for alternative energy production. It was noted that the main drawback of the modern expert system for assessing the energy properties of substances used as fuels is not taking into account the influence of the chemical bond type on its energy and energy characteristics of fuels in general. It was shown that the solution to this problem is possible through the use of the unified model of chemical bond of elements, which considers any chemical bond as an overlay (resonance) of a 100\% covalent bond with either metal or metal and ionic components, with a subsequent assessment of the effect of each of them on total energy of the mixed bond. This model is the fundamental basis of the System of chemical bonds and compounds (SCBC) in the form of the "Chemical Triangle". The possibility of using the "Chemical Triangle" as a modern intellectual basis for digital systematization and creation of a database of energy characteristics of various substances based on homo- and heteronuclear compounds of elements was shown. A computer database was developed to assess the complex impact of composition and chemical bond type on its energy characteristics, structure and properties of substances and materials.
\end{abstract}

\section{Introduction}

Chemical energy is the most important component of general energy $[1,2]$. It should also be noted that nuclear (about $13.5 \%$ of the world's energy production), wind, hydro-, solar and other types of energy also rely on chemical energy [1-3]. After all, the main types of materials (metals, organic and inorganic polymers, including ceramics, glasses, binders, etc.) that are needed for the implementation in practice [1-3] of these alternative methods of energy production are also obtained on the basis of chemical transformations of a substance associated with the release and consumption of energy upon breaking and forming chemical bonds. Moreover, wind and hydropower as a carrier of kinetic energy uses the Earth's atmosphere and hydrosphere in the form of chemicals based on $\mathrm{O}_{2}, \mathrm{~N}_{2}$ and $\mathrm{H}_{2} \mathrm{O}$ molecules. At the same time, due to the fragmentation of scientific knowledge at the second (differential) and the third (analytical-integral) stages of its development [4] (the number of scientific disciplines has exceeded 17000), this obvious fact of the unique practical significance of chemical energy is usually ignored. There is a need to address these omissions, including the revision of a number of traditional scientific paradigms and terms. It should be noted that the concept of "energy" is also interpreted in different ways today, including using traditional, but no longer accurate enough for the XXI century definitions. For example, energy (E) is a physical quantity that shows what work a body (or several bodies) can do, or the energy of a body.

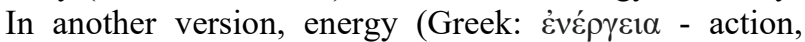
activity, force, power) is a scalar physical quantity that is a single measure of various forms of movement and interaction of matter, a measure of the transition of the matter movement from one form to another. First, in the fourth (integral-differential) stage of development of scientific knowledge and the paradigm of multi-level organization of substance and matter [4-6] it is doubtful to call any energy a "physical quantity" (in fact there are chemical and other types of energy). It is more correct to use a more precise and universal scientific concept of "material quantity". Secondly, energy is not just an abstract quantity that characterizes body's ability to work or a measure of various forms of motion and interaction of matter. Energy is an objective reality and one of the two most important characteristics, or properties, of matter as a whole, which determines, within the framework of the law of conservation of mass - energy and particle-wave dualism, the features of the modern paradigm of the multilevel structural organization of substance and matter as a whole [6]. Together with mass, energy determines the unity of the nature of various forms of existence of matter and at the same time, their

* Corresponding author: sirotkin-49@mail.ru 
differences, including the varieties of material objects in their elemental composition and their bond type, structure and properties (fields, substances, material bodies, etc.). At the same time, it is the field form of matter that is characterized by energy and wave characteristics, providing fundamental interactions of elements that make up specific varieties of material systems that are different in size and extent. Therefore, energy is a property of matter that characterizes the magnitude of internal force that bonds elements, which compose different levels of structural organization of matter in the form of closed physical systems (substances: atoms, molecules, cells, bodies, etc.), or their potential interactions, movement or transformation during intra-or inter-level contacts, including the kinetic energy of motion [5,6]. Next, one can evaluate the relationship of energy with various forms of motion and interaction of matter, what kind of work it can do, etc. As a result, it is necessary to distinguish between the energy concentrated inside the following types of material systems: fields, substances (nuclear and electromagnetic physical fields in an atom, chemical one in a molecule, etc.), material bodies (various fields in human body, the planet Earth, etc.) and megamaterial systems (the gravitational field in the Solar system, etc.).

The problem of improving the efficiency of assessing the energy properties of chemicals used as a source of energy is the most important and urgent task of modern science and industry. To solve this problem, we need a modern fundamental scientific base that provides systematic and comprehensive assessment of the impact on the energy characteristics of substances not only of the chemical composition, but also of the type of chemical bond of the elements. However, until recently, there was no fundamental scientific basis that would provide the possibility of a comprehensive systemic quantitative assessment of the energy characteristics of chemical bonds of compounds of elements (chemicals) [7]. In 1990-1992, the authors of this work managed for the first time to comprehensively assess the influence of the elemental composition and the type of bond (through the ratio of the covalent character, metallic character and ionic character) on structure and some properties of homo- and heteronuclear compounds of elements (chemicals) within the "Chemical triangle" (CT). Further, the CT developed and proved the predictive ability of these approaches using the characteristics of antimony - nitrogen bonds and the synthesis of a new class of resin-like inorganic oligomers [7]. Moreover, from 2000 to 2020, the understanding grew that the CT bears the features (Fig. 1) of a qualitatively new material system - the System of Chemical Bonds and Compounds (SCBC) $[1,3,8,10]$. Moreover, within the framework of a two-level view of the organization of matter in the form of the atomic-molecular theory (1860), we consider the SCBC and the CT [1,9] as an addition to the physical system of atoms in the form of the Periodic system (PS) of elements by D. I. Mendeleev (1869).

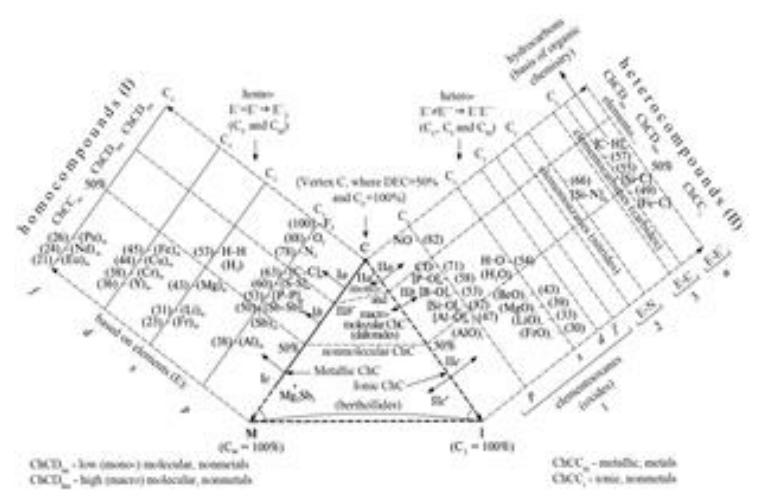

Fig. 1. CT is a system of chemical bonds and compounds (SCBC) that combine homo- and heteronuclear binary chemical compounds in the form of nonmolecular metallic, ionic, mono-, oligo-, and macromolecular compounds, along with polymer bodies where ChCDs are discrete chemical compounds (molecular) and ChCCs are continuous chemical compounds (nonmolecular).

The aim of this work is to substantiate and demonstrate the possibility of using the System of Chemical Bonds and Compounds (SCBC) in the form of a "Chemical triangle" (Fig.1) as a modern intellectual basis for the digital systematization of the energy characteristics of substances and the evaluation of their practical use as fuels.

\section{Methods}

At the first stage, it is necessary to evaluate the complex effect of elemental composition and type of mixed chemical bond in homo- and heteronuclear compounds of elements according to the unified quantum chemical model (Formula 1) on its energy.

$$
\Psi_{\mathrm{SE}}=\mathrm{C}_{1} \Psi_{\mathrm{A} \div \mathrm{B}}+\mathrm{C}_{2} \Psi_{\mathrm{A}+-\uparrow-\mathrm{B}+}+\mathrm{C}_{3} \Psi_{\mathrm{A}+\rightarrow \div \mathrm{B}-},
$$

where $\mathrm{A}$ and $\mathrm{B}$ are the nuclei or atomic cores of different elements in the respective heteronuclear chemical bond; $\mathrm{C}_{1}, \mathrm{C}_{2}$, and $\mathrm{C}_{3}$ are coefficients whose squares characterize the bond covalent character, metallic character, and ionic character $\left(\mathrm{C}_{\mathrm{C}}, \mathrm{C}_{\mathrm{M}}\right.$, and $\mathrm{C}_{\mathrm{I}}$ respectively), and whose sum is unity or $100 \% ; \Psi_{\mathrm{A} \div \mathrm{B}}$ is the covalent component in the total wave function of the shared (valence) electrons (SEs); $\Psi_{\mathrm{A}^{+-\uparrow-\mathrm{B}+}}$ is the metallic component in the total wave function of SEs; and $\Psi_{\mathrm{A}+\rightarrow \text { B- }}$ is the ionic component in the total wave function of SEs. For a homonuclear bond, the elements are the same, that is, there are A-A or B-B bonds, and Formula 1 is simplified by eliminating the last term, since there is no ionic component in these bonds. Methods of quantitative assessment of $\mathrm{C}_{C}, \mathrm{C}_{\mathrm{M}}$ and $\mathrm{C}_{\mathrm{I}}$ are given in $[1,10,11]$.

\section{Results and Discussion}

General nature of changes in energy of homonuclear bonds of the Periodic system elements, located on the left side of the CT system of elements (Fig. 1) upon 
moving from the vertex $\mathrm{C}$ to the vertex $\mathrm{M}$ is characterized by the data of Tables 1 and 2. It was found that with increasing $\mathrm{C}_{M}$ and decreasing $\mathrm{C}_{C}$ in the homobonds of elements forming metallic substances (Table 1) their energy is small and monotonically decreases, while in the case of transition from non-metallic substances, including diamond to metallic ones (Table 2) the bond energy is characterized by a higher value in non-metals, passing through the maximum of the carbon-carbon bond and falling further in metals. This allows us to conclude that in predominantly covalent (non-metallic) bonds, an increase in $C_{M}$ to the value of less than around $50 \%$ (Fig. 1) provides an increase in the bond energy, and in predominantly metallic bonds an increase in $C_{M}$ to more than $50 \%$ provides a monotonic decrease in it.

Table 1. Effect of the $\mathrm{C}_{M}\left(\mathrm{C}_{\mathrm{C}}\right)$ ratio in the homonuclear bonds of the elements of Group 1 of the PS, which form metals, on their energy.

\begin{tabular}{|c|c|c|}
\hline Bond & $\mathrm{C}_{M}\left(\mathrm{C}_{\mathrm{C}}\right) \%$ & Bond energy, $\mathrm{kJ} / \mathrm{mol}$ \\
\hline $\mathrm{Li}-\mathrm{Li}$ & $76.6(23.4)$ & 111 \\
\hline $\mathrm{Na}-\mathrm{Na}$ & $77.5(22.5)$ & 75 \\
\hline $\mathrm{K}-\mathrm{K}$ & $79.3(21.7)$ & 51 \\
\hline $\mathrm{Rb}-\mathrm{Rb}$ & $80.5(19.5)$ & 48 \\
\hline $\mathrm{Cs}-\mathrm{Cs}$ & $81.4(18.6)$ & 45 \\
\hline
\end{tabular}

Table 2. Influence of the $\mathrm{C}_{M}\left(\mathrm{C}_{C}\right)$ ratio in the homonuclear bonds of elements of the 2nd Period of the PS, which form nonmetals and metals, on their energy.

\begin{tabular}{|c|c|c|}
\hline Bond & $\mathrm{C}_{\mathrm{M}}\left(\mathrm{C}_{\mathrm{C}}\right), \%$ & $\begin{array}{c}\text { Bond energy, } \\
\mathrm{kJ} / \mathrm{mol}\end{array}$ \\
\hline $\mathrm{F}-\mathrm{F}$ & $0(100)$ & 138 \\
\hline $\mathrm{O}-\mathrm{O}$ & $10.6(89.4)$ & 146 \\
\hline $\mathrm{N}-\mathrm{N}$ & $21.2(78.8)$ & 160 \\
\hline $\mathrm{C}-\mathrm{C}$ & $31.6(68.4)$ & 348 \\
\hline $\mathrm{B}-\mathrm{B}$ & $49.0(51.0)$ & 335 \\
\hline $\mathrm{Be}-\mathrm{Be}$ & $63.3(36.7)$ & 223 \\
\hline $\mathrm{Li}-\mathrm{Li}$ & $76.6(23.4)$ & 111 \\
\hline
\end{tabular}

For heteronuclear bonds, there is a similar effect of the ratio, this time of three components, of the chemical bond $\left(\mathrm{C}_{\mathrm{C}}, \mathrm{C}_{\mathrm{M}}\right.$, and $\left.\mathrm{C}_{\mathrm{I}}\right)$. For example, in a series of oxides of sulfur, phosphorus, silicon, boron, aluminium, sodium $[1,11]$ in CT when moving top-down from the vertex M to the vertex I, first there is an increase of the elementoxygen bond energy (in $\mathrm{kJ} / \mathrm{mol}$ the values are: 298,369 , $444,499,423,84)$ accompanied by the reduction of $\mathrm{C}_{\mathrm{C}}$ and the increase of $\mathrm{C}_{M}$ and $\mathrm{C}_{\mathrm{I}}$ of the E-O bond, then goes through a maximum in boron oxide (499) and then drops (84) in sodium oxide. However, in general, the energy of heteronuclear bonds is significantly higher than the energy of homonuclear bonds, which makes substances based on the former more preferable as potential energy carriers for their use as fuels. The authors and the school developed under their leadership created two computer programs $[16,17]$, which represent a database for digital systematization of assessing the impact of chemical nature of a substance on energy characteristics of bonds in substances, their structure and properties.

At the second stage, it is important to determine how to efficiently extract the chemical bond energy concentrated in various substances based on homo- or heteronuclear compounds of elements. Traditionally, their ability to undergo exothermic chemical reactions upon the interaction between chemical compounds is of particular importance for evaluating the use of certain substances as fuel. The possibility of such reactions is determined by the difference in bond energy of starting substances and the reaction products. This includes their interaction with oxygen (oxidation reactions, including burning or explosion, when the maximum amount of energy is released). In [2], we were able to show for the first time that the probability of an exothermic effect (when heat is released) increases with the increase of the difference $\Delta \mathrm{C}_{\mathrm{C}}\left(\Delta \mathrm{C}_{\mathrm{M}}\right)$ of bonds in the starting chemicals when they are converted into heteronuclear reaction products. Also, for the first time, the boundary of the transition (Er) of halide formation reactions from the exo- to the endothermic type was clarified. It is approximately equal to $12 \Delta \mathrm{C}_{\mathrm{M}}$ units. At $\Delta \mathrm{C}_{\mathrm{M}}>12$ the reaction is exothermic, at $\Delta \mathrm{C}_{\mathrm{M}} \approx 12$ equilibrium occurs at the Er point, and at $\Delta \mathrm{C}_{\mathrm{M}}$ below 12 , the reaction proceeds with heat absorption (endoeffect). In the case of interaction of homonuclear compounds $\left(\mathrm{O}_{2}, \mathrm{H}_{2}\right.$, etc.), it can be considered established that with an increase in the difference between $C_{C}$ and $C_{M}$ in them, their reactivity increases with a simultaneous increase in the thermal effect of the reaction. In the case of heteronuclear compounds, such as ammonia and methane, the difference in their composition and type of bond leads to the fact that methane interacts more easily with $\mathrm{O}_{2}$. This is due to a decrease in its thermal effect of the reaction (heat of combustion), which explains the lower reactivity of $\mathrm{NH}_{3}$ compared to $\mathrm{CH}_{4}$, and the lower exothermicity of the ammonia combustion reaction.

Today, SCBC in the form of CT (Fig.1) is developed by the authors as a modern fundamental scientific and new methodological basis for the systematic study of the influence of the compounds' chemical nature on the bonds' characteristics (the nature of the distribution of electron density between elements, length and energy), structure (low-, oligo-, high-molecular and nonmolecular metal and ionic, etc.) and properties of various substances and materials [1-4, 8-17]. We should especially note the increased interest in such studies abroad and in the Russian Federation, including the use of our methodology and methods for calculating the components of mixed types of chemical bonds in steels, aluminum alloys, etc. [9, 18-25].

\section{Conclusion}

The need to understand the role of the chemical form of matter that accumulates the most affordable part of the energy on the Earth, as the most important component of the overall energy system, is noted. The possibility of using the System of Chemical Bonds and Compounds (SCBC) in the form of a "Chemical triangle" as a modern intellectual basis for the digital systematization of the energy characteristics of chemicals and the evaluation of their practical use as fuels was justified. On this basis, two computer programs were created, which represent a database for digital systematization of the 
impact of the chemical nature of a substance on the energy characteristics of bonds in it. For the first time, within the framework of the "Chemical triangle", it was shown that the nature of the energy change of chemical bond in homo- A-A or B-B and heteronuclear A-B bonds, when moving from vertex $\mathrm{C}$ to vertices $\mathrm{M}$ and $\mathrm{I}$ passes through a maximum value at intermediate energy (not maximum) values of CC, CM and CI. It was shown that the possibility of chemical reactions is determined by the difference in the ratio of $\mathrm{CC}, \mathrm{CM}$ and $\mathrm{CI}$, and bond energies of the starting substances and reaction products; the interval of $\triangle \mathrm{CM}$, which determines the possibility of a reaction with heat release, was defined.

\section{References}

1. O.S. Sirotkin, R.O. Sirotkin, Chemistry (Principles of a Single Chemistry) (KNORUS, Moscow, 2017) (in Russian)

2. O.S. Sirotkin, A.M. Pavlova, R.O. Sirotkin et all, E3S Web of Conferences, 124, 01017 (2019)

3. O.S. Sirotkin, Principles of Innovative Materals Science (INFRA-M, Moscow, 2020) (in Russian)

4. V.I. Kuznetsov, G.M. Idlis, V.N. Gutina, Natural Science (Agar, Moscow, 1996) (in Russian)

5. O.S. Sirotkin, System of Universe (Scientific principles of the contemporary view of the world) (Ruscience, Moscow, 2020) (in Russian)

6. O.S. Sirotikin, Integral-differential principles of unitary natural science (Paradigm of multi-level organization of matter as a natural basis for diversity and unity of nature of objects of the universe system) (KSPEU, Kazan, 2011) (in Russian)

7. R.O. Sirotkin, Physicochemistry of Homo- and Heteronuclear Binary Substances and Materials Based on Them, Rusains, Moscow (2018) (in Russian)

8. O.S. Sirotkin, International Conference "Chemical Education and Development of the Society", Mendeleev University of Chemical Technology of Russia, Moscow (2000) (in Russian)

9. R.O. Sirotkin, O.S. Sirotkin, Russ. J. Phys. Chem. A, 94, 1153 (2020)

10. O.S. Sirotkin, R.O. Sirotkin, A.M. Trubacheva, Russ. J. of Inorg. Chem., 50, 67 (2005)

11. O.S. Sirotkin, R.O. Sirotkin, A.M. Trubacheva, Characteristics of homo- and heteronuclear bonds of fine electronic-nuclear structure and their impact on properties of metallic and nonmetallic materials (KSPEU, Kazan, 2009) (in Russian)

12. O.S. Sirotkin, R.O. Sirotkin, P.B. Shibaev, Russ. J. Inorg. Chem., 56, 1104 (2011)

13. Sirotkin R.O., Sirotkin O.S., Ivshin I.V. et all, ARPN J. of Eng. and Appl. Sci., 11, 9664 (2016)

14. R.O. Sirotkin, O.S. Sirotkin, IOP Conf. Series: Materials Science and Engineering, 327, 042119 (2018)
15. R.O. Sirotkin, O.S. Sirotkin, Materials Science Forum, 992, 952 (2020)

16. O.S. Sirotkin, P.A. Maloletkov, System of homonuclear substances, Certificate of the state registration of the program for PC, 2014617629 (29 July 2014)

17. O.S. Sirotkin, R.O. Sirotkin, A.M. Pavlova, et all, Chemical bond characteristics and their impact on structure and properties of simple substances and oxides, Certificate of the state registration of the program for PC, 2019618435 (1 July 2019)

18. W.B. Jensen, J. Chem. Educ., 86, 278 (2009)

19. G.D. Sproul, J. Phys. Chem., 98, 6699 (1994)

20. G.D. Sproul, J. Phys. Chem., 98, 13221 (1994)

21. L.C. Allen, J. Am. Chem. Soc., 144, 1510 (1992)

22. W.B. Jensen, J. Chem. Educ., 72, 395 (1995)

23. M.R. Leach, Found. Chem., 15, 13 (2013)

24. G.K. Moiseev, A.L. Ivanovskii, Russ. J. Phys. Chem. A., 81, 1203 (2007)

25. F. Kong, J. Mol. Struct., 90, 17 (1982) 